\title{
Foreword to Guidelines and Good Clinical Practice Recommendations for Contrast Enhanced Ultrasound (CEUS) in the Liver - Update 2012
}

\author{
L. D. Greenbaum
}

Affiliation

Winnie Palmer Hospital for Women \& Babies Orlando, The Hughes Center for Fetal Diagnostics, Orlando, FL, USA

Bibliography

DOI http://dx.doi.org/10.1055/

s-0032-1330486

Ultraschall in Med 2013; 34: 7

(c) Georg Thieme Verlag KG

Stuttgart · New York .

ISSN 0172-4614

\section{Correspondence}

Lennard D. Greenbaum

Winnie Palmer Hospital for

Women \& Babies Orlando,

The Hughes Center for Fetal

Diagnostics

83 West Miller Street

Orlando, FL 32806

USA

lengreenbaum@me.com

Tel.: ++ 1/407/6496910
The European Federation of Societies for Ultrasound in Medicine and Biology (EFSUMB) deserves our thanks for recognizing the need for ultrasound contrast agent (UCA) guidelines and for writing and publishing them in 2004 [1] and 2008 [2]. The third iteration of UCA guidelines in the liver, Guidelines and Good Clinical Practice Recommendations for Contrast Enhanced Ultrasound (CEUS) in the Liver - Update 2012, contains major changes from the two previous versions. In the 2012 guideline, EFSUMB acknowledges the almost worldwide acceptance and usage of CEUS in the liver. A major change is the fact that this new guideline is a collaborative document with the participation of World Federation for Ultrasound in Medicine and Biology (WFUMB), Asian Federation of Societies for Ultrasound in Medicine and Biology (AFSUMB), American Institute of Ultrasound in Medicine (AIUM), Australasian Society for Ultrasound in Medicine (ASUM) and International Contrast Ultrasound Society (ICUS). This is the first time that experts from around the world in the non-cardiac use of CEUS have come together to create a unified guideline on its use. This international participation gives the document more strength and proclaims the worldwide recognition of the use of CEUS to image the liver.

The 2012 guideline begins with a most appreciated "List of Abbreviations," which provides easy access for reference. There also is a new section on "Terminology," which includes contributordefined descriptive terms that should be used when reporting and publishing CEUS findings, including a newer contrast agent that has a postvas- cular phase. Having worldwide agreement on terminology greatly improves communication of results. Utilizing this terminology, a most significant change in this guideline is a marked expansion of the description of various liver lesions and how they appear on contrast-enhanced images. The section on Safety Considerations has been brought up to date citing several new papers confirming the excellent safety profile of UCAs. The bulk of the guideline is divided into sections dealing with characterization of focal liver lesions (FLL) (subdivided into noncirrhotic and cirrhotic livers), characterization of portal vein thrombosis, the use of CEUS when performing a biopsy, detection of FLL, using CEUS intraoperatively, monitoring ablation treatment, evaluating liver transplants and monitoring systemic treatment of malignancies.

This guideline should be a major reference for anyone performing CEUS of the liver. Hopefully, here in the United States, we will have approval by our Food and Drug Administration in the foreseeable future and will be able to put this excellent guideline to use.

\section{References}

1 Albrecht T, Blomley M, Bolondi L et al. Guidelines for the use of contrast agents in ultrasound. January 2004. Ultraschall in Med 2004; 25: 249-256

2 Claudon $M$, Cosgrove D, Albrecht $T$ et al. Guidelines and good clinical practice recommendations for contrast enhanced ultrasound (CEUS) - update 2008. Ultraschall in Med 2008; 29: 28-44 